\title{
Shape and Area of Keratocytes Are Related to the Distribution and Magnitude of Their Traction Forces
}

\author{
Ayane Sonoda, Chika Okimura, and Yoshiaki Iwadate* \\ Faculty of Science, Yamaguchi University, Yamaguchi 753-8512, Japan
}

\begin{abstract}
Fish epidermal keratocytes maintain an overall fan shape during their crawling migration. The shape-determination mechanism has been described theoretically and experimentally on the basis of graded radial extension of the leading edge, but the relationship between shape and traction forces has not been clarified. Migrating keratocytes can be divided into fragments by treatment with the protein kinase inhibitor staurosporine. Fragments containing a nucleus and cytoplasm behave as mini-keratocytes and maintain the same fan shape as the original cells. We measured the shape of the leading edge, together with the areas of the ventral region and traction forces, of keratocytes and mini-keratocytes. The shapes of keratocytes and mini-keratocytes were similar. Mini-keratocytes exerted traction forces at the rear left and right ends, just like keratocytes. The magnitude of the traction forces was proportional to the area of the keratocytes and mini-keratocytes. The myosin II ATPase inhibitor blebbistatin decreased the forces at the rear left and right ends of the keratocytes and expanded their shape laterally. These results suggest that keratocyte shape depends on the distribution of the traction forces, and that the magnitude of the traction forces depends on the area of the cells.
\end{abstract}

Key words: cell locomotion, cell migration, focal adhesions, myosin II, stress fibers

\section{Introduction}

Cell-crawling migration plays essential roles in complex biological phenomena, including development (Lauffenburger and Horwitz, 1996; Ridley et al., 2003), wound healing (Martin, 1997), immune system function (Parent, 2004), and cancer metastasis (Friedl and Alexander, 2011). Crawling cells have characteristic shapes that depend on the cell type (Mogilner and Keren, 2009). How the shape is determined in the absence of hard cell walls is an interesting question. Fish epidermal keratocytes maintain an overall fan shape during their crawling migration and are used widely to study shape-determination mechanisms.

Migration of keratocytes with a constant fan shape has been described geometrically by the graded radial extension model (Lee et al., 1993). The principal concept of this model is that radial expansion of the leading edge perpendicular to the cell margins occurs in a graded manner. As a molecular basis of the radial expansion of the leading edge, graded expansion rate of the leading edge depends on the graded density of the filamentous actin (F-actin) there (Keren et al., 2008). Actin polymerization at the leading

\footnotetext{
*To whom correspondence should be addressed: Yoshiaki Iwadate, Faculty of Science, Yamaguchi University, Yamaguchi 753-8512, Japan. Tel/Fax: +81-83-933-5760

E-mail: iwadate@yamaguchi-u.ac.jp
}

edge pushes the membrane forward and, consequently, the polymerized $\mathrm{F}$-actin moves backward with respect to the substrate by the reaction force from the membrane (Forscher and Smith, 1988). This backward movement of F-actin is termed retrograde F-actin flow (RF) (Gupton and Waterman-Storer, 2006; Katoh et al., 1999a, 1999b; Vallotton et al., 2005). The graded RF rate is correlated with the graded expansion rate of the leading edge (Barnhart et al., 2011). Interestingly, the graded RF rate seems to be regulated by the strength of adhesion of the cell to the substrata.

Crawling cells exert traction forces on the substratum via focal adhesions (Harris et al., 1980, 1981). The focal adhesions are matured by these mechanical forces (Galbraith et al., 2002). As a result, the adhesion strength should increase. The traction forces may thus regulate cell shape by regulating the adhesion strength and the graded RF rate. Moreover, the traction forces may directly change the cell shape, because the geometry of the soft membrane is regulated by the mechanical force-balance on it. In fact, in Vshaped fibroblasts, traction forces are actively generated near the leading edge (Beningo et al., 2001; Dembo and Wang, 1999; Munevar et al., 2001a, 2001b, 2004). In contrast, in fan-shaped keratocytes, these forces are generated at the rear left and right ends (Burton et al., 1999; Chen et al., 2013; Doyle et al., 2004; Fournier et al., 2010; Jurado et al., 2005). These findings suggest that cell shape may be 
regulated by the distribution of traction forces.

The relationship between traction forces and cell size is another interesting issue. The maximum force of large fibroblasts is several tens of kilopascals (Dembo and Wang, 1999; Munevar et al., 2001a, 2001b), although that of small cell types, such as Dictyostelium cells (Iwadate and Yumura, 2008a; Lombardi et al., 2007; Tanimoto and Sano, 2014), is several kilopascals or smaller. The magnitude of the traction forces may be related to cell size.

Migrating keratocytes are rarely divided into fragments spontaneously in culture (Euteneuer and Schliwa, 1984). Fragmentation can be also induced by treatment with the protein kinase inhibitor staurosporine (Verkhovsky et al., 1999). Fragments containing nucleus and cytoplasm behave as downsized mini-keratocytes. In this study, we measured the shape of the leading edge, the areas of the cell-ventral region, traction forces, and the distribution of vinculin (a kind of focal adhesion protein) in keratocytes and minikeratocytes. The shapes of the leading edges in keratocytes and mini-keratocytes were similar. Mini-keratocytes, like keratocytes, exerted traction forces at the rear left and right ends. The myosin II ATPase inhibitor blebbistatin decreased the forces at the rear left and right ends of the keratocytes and expanded their shape laterally. The magnitude of the traction forces and the area of focal adhesions, which acted as scaffolds for the forces, were proportional to the area of the keratocytes and mini-keratocytes. These results suggest that keratocyte shape depends on the distribution of traction forces and that the magnitude of the traction forces depends on the area of the cells.

\section{Materials and Methods}

\section{Cell culture}

Keratocytes from the scales of Central American cichlid (Theraps nicaraguense) were cultured as described previously (Mizuno and Sekiguchi, 2011; Mizuno et al., 2003; Tsugiyama et al., 2013). Briefly, fish scales were extracted and washed in culture medium (Leibovitz's medium; L-15, L5520; Sigma-Aldrich, St Louis, MO) supplemented with $10 \%$ fetal calf serum (Nichirei, Tokyo, Japan) and antibiotic/antimycotic solution (09366-44; Nacalai Tesque, Kyoto, Japan). The scales were placed external side up on the bottom of a chamber, the bottom of which was made of a coverslip, then covered with another small coverslip and allowed to adhere to the bottom coverslip for $1 \mathrm{~h}$ at $37^{\circ} \mathrm{C}$. Then, after removal of the upper coverslip, culture medium was added to the chamber and the scales were kept at $37^{\circ} \mathrm{C}$ again overnight to allow the cells to spread from the scale. Cells were washed briefly with Dulbecco's phosphate-buffered saline without $\mathrm{Ca}^{2+}$ and $\mathrm{Mg}^{2+}$ (PBS--) and then treated with $0.5 \mathrm{~g} / \mathrm{L}$ trypsin and $0.53 \mathrm{mM}$ EDTA in PBS (trypsin-EDTA, 32778-34; Nacalai Tesque) for 30 to $60 \mathrm{~s}$. The trypsin was quenched with a 10 -fold excess of culture medium.

\section{Fragmentation of keratocytes}

Staurosporine (569397; Calbiochem, San Diego, CA) was dissolved at $10 \mu \mathrm{M}$ in DMSO and then diluted 100 times with culture medium. This staurosporine medium was applied to the chamber, to the bottom of which the cells had adhered, just after removal of the culture medium. After $1 \mathrm{~h}$, almost all of the cells were fragmented. The cell fragments were washed with culture medium and used for experiments.

\section{Fixed-cell staining}

Fixed-cell staining was performed according to methods described previously (Miyoshi and Adachi, 2012; Okeyo et al., 2009), with small modifications. Briefly, cells were fixed with $4 \%$ paraformaldehyde for $15 \mathrm{~min}$, permeabilized with $0.1 \%$ Triton X-100 for 10 min, and blocked with $0.2 \%$ gelatin for $30 \mathrm{~min}$. The cells were then incubated with primary antibody, namely mouse monoclonal vinculin (1:800 dilution, V9131, Sigma-Aldrich) or Alexa Fluor 488 phalloidin (0.33 U/mL, A12379; Life Technologies, Carlsbad, $\mathrm{CA}$ ), or both, for $60 \mathrm{~min}$. After several washes with $0.2 \%$ gelatin, the cells were incubated with secondary antibody, namely Alexa Fluor 546 Anti-mouse IgG (1:2,000 dilution, A-11030, Life Technologies), for $60 \mathrm{~min}$. Fixation and staining were all performed at room temperature. For myosin IIA staining, the cells were permeabilized with $0.02 \%$ Triton X-100. Rabbit polyclonal myosin IIA (1:200 dilution, M8064, Sigma-Aldrich) and Alexa Fluor 546 Anti-rabbit IgG (1:2,000 dilution, A-11071, Life Technologies) were used as the primary and secondary antibody, respectively.

\section{Microscopy}

Fluorescence images of live and fixed cells were detected under an inverted microscope (Ti; Nikon, Tokyo, Japan) equipped with a laser confocal scanner unit (CSU-X1; Yokogawa, Tokyo, Japan) and an EM CCD camera (DU897; Andor, Belfast, UK) by using a 100× objective lens (CFI Apo TIRF 100× H/1.49; Nikon, Tokyo, Japan).

\section{Traction force microscopy}

Traction force microscopy was performed according to the methods described previously (Iwadate and Yumura, 2008a, 2008b) with small modifications. A type of polydimethylsiloxane (CY52-276A and B; Dow Corning Toray, Tokyo, Japan) was used as the material for the elastic sheet. CY52-276A and B were mixed at 6:10 in weight. A 40- to 45-mg aliquot of the mixture was spread on a $22 \times 22 \mathrm{~mm}$ coverslip (No. 0, Matsunami, Osaka, Japan). After solidification of the mixture, the solidified substrata were kept in a hermetically sealed case with a $50-\mu \mathrm{L}$ aliquot of liquid silane (3-aminopropyl triethoxysilane, Sigma-Aldrich) at $70^{\circ} \mathrm{C}$ for $1 \mathrm{~h}$, in order to attach the silane to the surface of the silicone substrata by vapor deposition. A round chamber $(16 \mathrm{~mm}$ in diameter $\times 2 \mathrm{~mm}$ in depth) was then assembled, using the coated coverslip to form the bottom of the chamber. Red fluorescent 
carboxylate-modified microspheres $(20 \mathrm{~nm}$ in diameter; peak excitation and emission wavelengths 580 and $605 \mathrm{~nm}$ respectively; F-8786, Life Technologies) were attached to the surface of the substrata by means of binding between the amino group in the silane and the carboxyl group in the microspheres.

Young's moduli of the elastic substrata were measured by the method of Lo and colleagues (Lo et al., 2000). Briefly, a steel ball $\left(0.5 \mathrm{~mm}\right.$ diameter, $\left.7.8 \mathrm{~kg} / \mathrm{m}^{3}\right)$ was placed on a substratum embedded with fluorescent beads. Young's modulus was calculated as $Y$ $=3\left(1-p^{2}\right) f / 4 d^{3 / 2} r^{1 / 2}$, where $f$ is the force exerted on the sheet, $d$ is the indentation, $r$ is the radius of the steel ball, and $p$ is the Poisson ratio [assumed to be 0.5 (Dembo et al., 1996)]. A typical value of Young's modulus was $13.8 \mathrm{kPa}$.

The image data were analyzed by using ImageJ (http:// rsbweb.nih.gov/ij). Images of fluorescent beads were first aligned to correct experimental drift by using the ImageJ plug-in StackReg. The displacement field and the traction force field were then calculated by using two plug-ins, PIV and FTTC (Tseng et al., 2012). The regularization parameter was set at $3 \times 10^{-10}$ for all traction force reconstructions.

\section{Measurements of migration velocities}

Migrating keratocytes or mini-keratocytes were observed under phase contrast microscopy using an inverted microscope (TS100; Nikon, Tokyo, Japan) with a $10 \times$ objective lens (CFI 10×; Nikon). Images taken with a CMOS camera (DMK41BUC02; Argo, Osaka, Japan) were transferred to a PC at 30-s intervals. The data were then analyzed using ImageJ with two plug-ins: Manual Tracking, and the Chemotaxis and Migration Tool.

To compare the migration velocities between keratocytes and mini-keratocytes, the cells treated with only $1 \%$ DMSO without staurosporine were used as control keratocytes.

\section{Blebbistatin treatment}

( \pm )-Blebbistatin (13186; Cayman, Ann Arbor, MI) was dissolved at $100 \mathrm{mM}$ in DMSO and then diluted 4,000 times with culture medium. This blebbistatin medium was then added to the chamber, to the bottom of which the cells had adhered, just after removal of the culture medium. After $15 \mathrm{~min}$, the cells in the chamber were used for experiments without removal of the blebbistatin medium.

\section{Correlation coefficient}

The correlation coefficient $(r)$ in Fig. 2E and 3D was calculated as follows:

$$
r=\frac{\sum_{i=1}^{n}\left(x_{i}-\bar{x}\right)\left(y_{i}-\bar{y}\right)}{\sqrt{\sum_{i=1}^{n}\left(x_{i}-\bar{x}\right)^{2}} \sqrt{\sum_{i=1}^{n}\left(y_{i}-\bar{y}\right)^{2}}}
$$

where $x_{i}$ and $y_{i}$ are variables and $\bar{x}$ and $\bar{y}$ are averages of $x_{i}$ and $y_{i}$, respectively.

\section{Results}

\section{Shape of leading edges in keratocytes and mini- keratocytes}

First, we analyzed the shape of the leading edges in keratocytes (Fig. 1A; typical image from 121 samples) and minikeratocytes (Fig. 1B; typical image from 97 samples). To compare the shapes of the leading edge quantitatively, we defined the "curvature radius" and "center angle" of the leading edge (Fig. 1C). A circular arc was traced along the leading edge of the cell. The two points at which the arc departed from the leading edge were connected to the center ( $o$ in Fig. 1C) of the arc by straight lines. The lengths of the two lines and the angle between them were defined as the "curvature radius" and "center angle," respectively.

Keratocytes migrate with little fluctuation of their fan shape (Barnhart et al., 2010, 2011). We therefore randomly selected bilaterally symmetric keratocytes from images of all the migrating keratocytes and measured their curvature radii and center angles (Fig. 1D and $1 F$ ). Those of the minikeratocytes were measured in the same way (Fig. 1E and $1 \mathrm{G})$. The average curvature radius of the keratocytes was significantly larger than that of the mini-keratocytes (Fig. $1 \mathrm{H})$. In contrast, there was no significant difference in the average center angle between the keratocytes and the minikeratocytes (Fig. 1I). These results indicated that the shape of the leading edge in mini-keratocytes was similar to that in the original keratocytes, even though the minikeratocytes were smaller.

\section{Traction forces and migration velocities of keratocytes and mini-keratocytes}

To test whether or not the fan shape of the keratocytes and mini-keratocytes was determined by the distribution of traction forces, we compared the distribution and magnitude of traction forces between keratocytes (Fig. 2A) and mini-keratocytes (Fig. 2C). The keratocytes exerted traction forces mainly at the rear left and right ends (Fig. 2B; typical pseudo-color image from 58 samples), as has been found in previous studies (Burton et al., 1999; Chen et al., 2013; Doyle et al., 2004; Fournier et al., 2010; Fuhs et al., 2014; Jurado et al., 2005). As expected, the minikeratocytes also exerted traction forces mainly at the rear left and right ends (Fig. 2D; typical pseudo-color image from 31 samples).

If keratocyte area were related to traction forces, then one would expect not the distribution of the forces but their magnitude to depend on the area of the cell. We measured traction forces from snapshot live images of randomlyselected locomoting keratocytes or mini-keratocytes which showed bilateral symmetry. The average values of maximum forces at the left and right trailing ends were calculated. Then, the relationship between the area of the ventral 

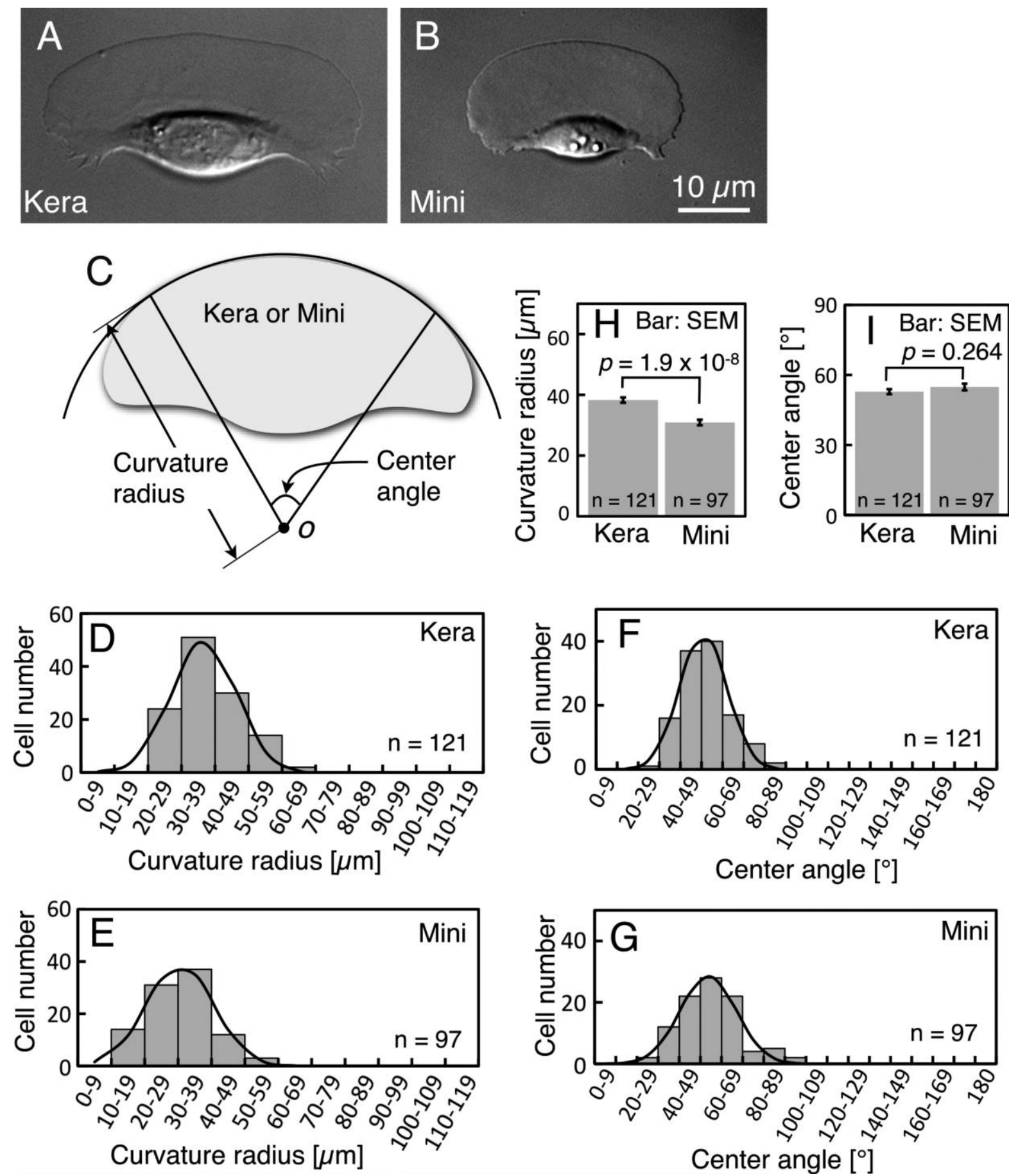

Fig. 1. Shapes of keratocytes and mini-keratocytes. (A) DIC image of a keratocyte. (B) DIC image of a mini-keratocyte. Images A and B are typical images from 121 and 97 samples, respectively. (C) Definition of "curvature radius" and "center angle" of the leading edge. See text for details. (D and E) Frequency distributions of curvature radius of keratocytes (D) and mini-keratocytes (E). (F and G) Frequency distributions of center angle of keratocytes (F) and mini-keratocytes $(\mathrm{G})$. (H) Averaged curvature radius of keratocytes (left) and mini-keratocytes (right). (I) Averaged center angle of keratocytes (left) and mini-keratocytes (right). 

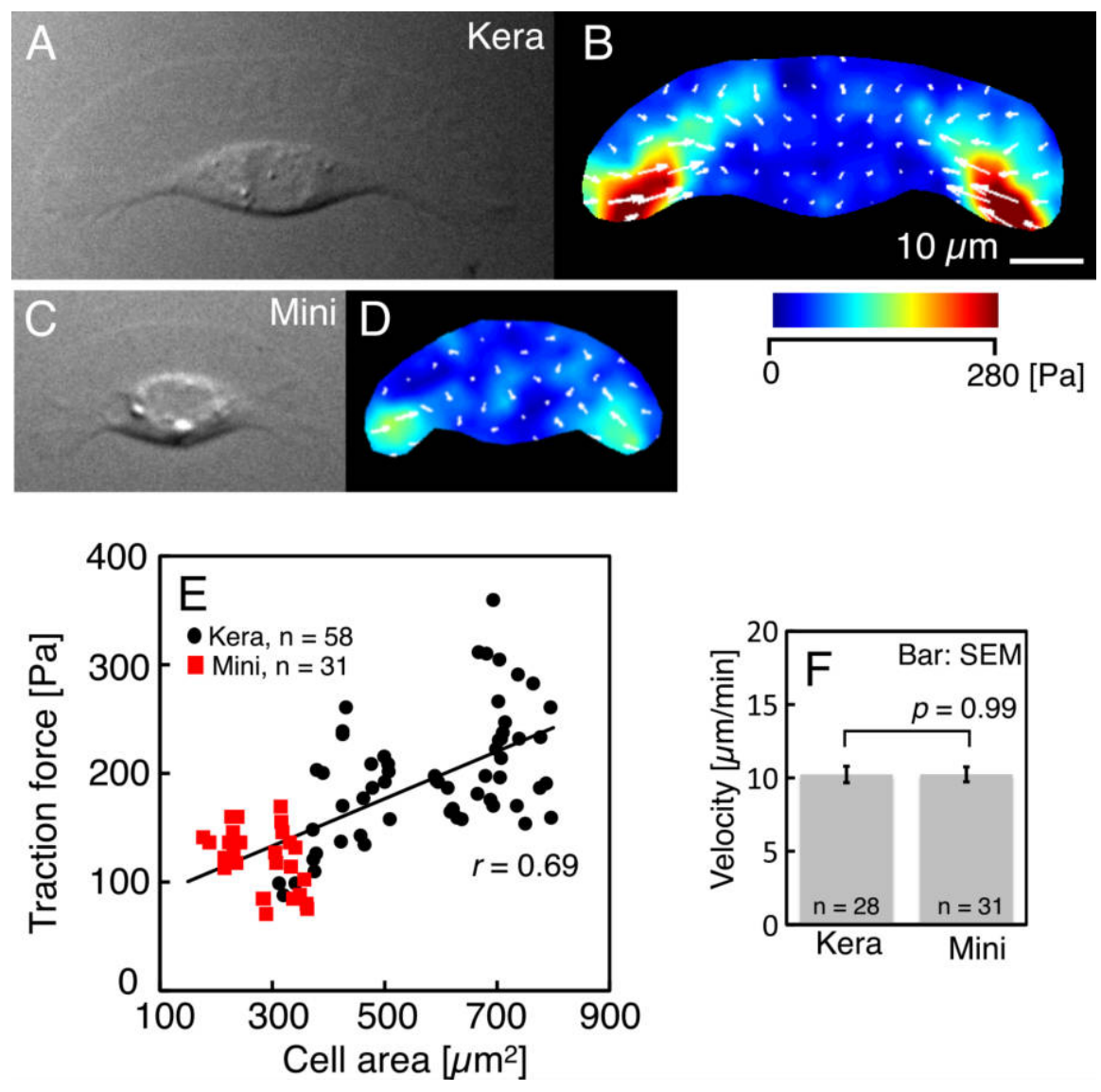

Fig. 2. Traction forces exerted by keratocytes and mini-keratocytes. (A) DIC image of a keratocyte. (B) Traction forces of the keratocyte in A. (C) DIC image of a mini-keratocyte. (D) Traction forces of the mini-keratocyte in C. Direction and length of white arrows in B and D respectively indicate the direction and relative magnitude of traction forces at the base of the arrows. Images A and B, and C and D, are typical images from 58 and 31 samples, respectively. (E) Relationship between the area of the ventral region of keratocytes (black circles) or mini-keratocytes (red squares) and the maximum traction force. Correlation coefficient $(r)$ was 0.69 . Straight line is a regression line obtained by the least squares method. (F) Migration velocities of keratocytes (left) and mini-keratocytes (right).

region of keratocytes or mini-keratocytes and the average traction force (Fig. 2E). The correlation coefficient $(r)$ between force and area was 0.69: as expected, there was a positive correlation between the area of keratocytes and mini-keratocytes and the maximum traction force.

Traction forces should play an important role in migration. To test whether the force of keratocytes and minikeratocytes reflects their migration, we compared the migration velocities of keratocytes and mini-keratocytes (Fig. 2F). Mini-keratocytes whose traction force was smaller than that of keratocytes migrated as fast at keratocytes, suggesting that migration velocity should depend on not traction force itself but traction force per the area of the cell.

\section{Molecular basis of traction forces of keratocytes and mini-keratocytes}

To test whether the molecular basis of the traction forces was identical regardless of the area of the cell, we first visualized the distributions of vinculin in keratocytes and mini-keratocytes by using immunofluorescent staining (Fig. $3 \mathrm{~A}$ and $3 \mathrm{~B}$; typical images from 59 and 44 samples, respectively). In the case of keratocytes, in agreement with the findings of previous studies (Barnhart et al., 2011; Doyle and Lee, 2005), vinculin was localized at the rear left and right ends (green arrows in Fig. 3A), where large traction forces were detected (compare Fig. 2B and Fig. 3A). In the mini-keratocytes, vinculin was localized at the same rear left and right ends (green arrows in Fig. 3B). The relative fluorescence intensities of vinculin (Fig. 3C) were calculated for the keratocyte in Fig. 3A and the mini-keratocyte in Fig. 3B by dividing the intensity at each point ( $a$ in Fig. 3C 

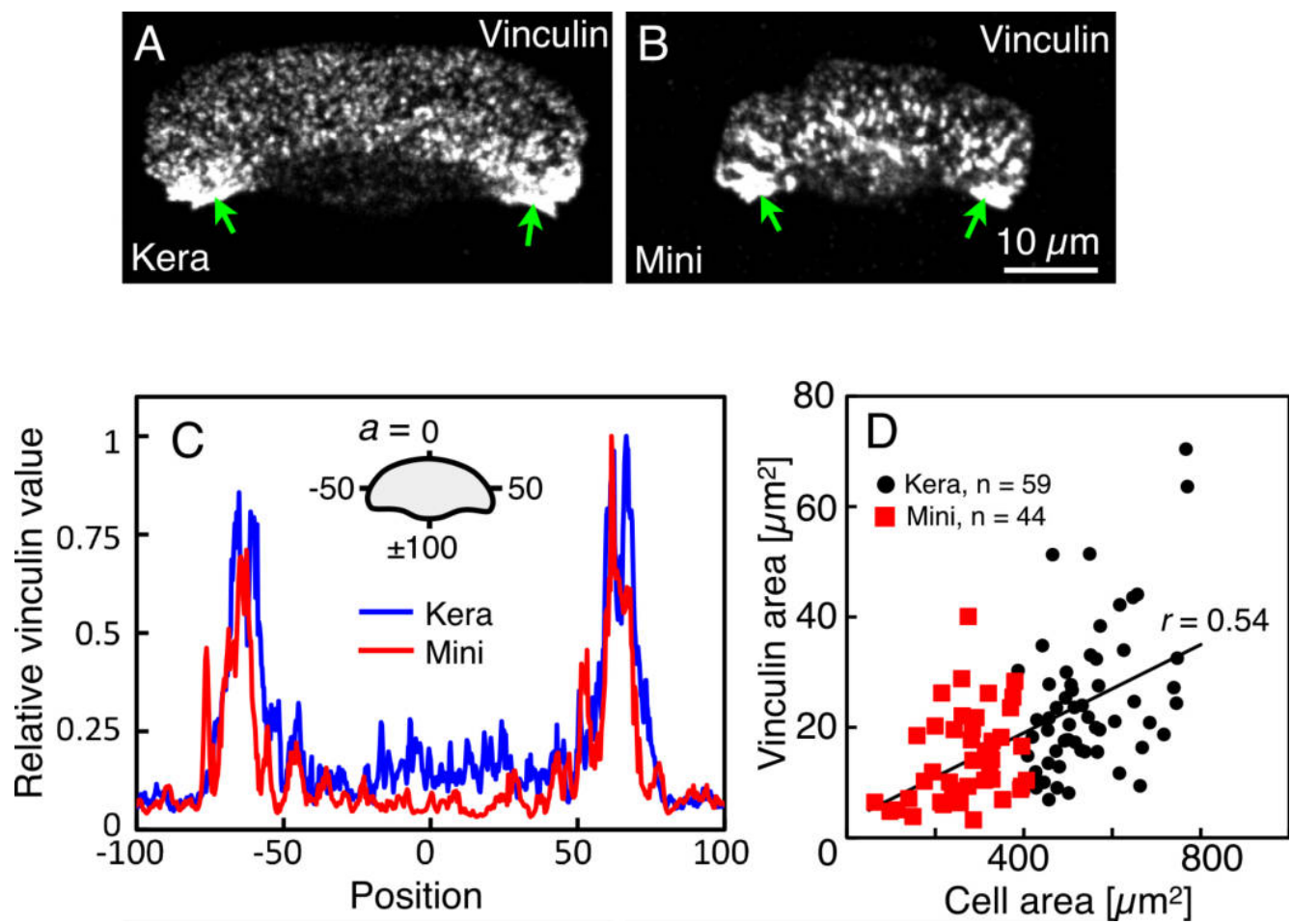

Fig. 3. Distribution of vinculin in keratocytes and mini-keratocytes. (A and B) Immunofluorescence staining of vinculin in a keratocyte (A) and a minikeratocyte (B). Images A and B are typical images from 59 and 44 samples, respectively. Green arrows indicate localized vinculin. (C) Relative fluorescence intensities of vinculin in the keratocyte (blue; obtained from A) and the mini-keratocyte (red; obtained from B). The values were calculated by dividing the intensity at each point ( $a$ in inset) around the cell perimeter by the maximum intensity of the cell. (D) Relationship between the area of the ventral region of the keratocyte (black circles) or mini-keratocyte (red squares) and the area of rear focal adhesions. Correlation coefficient $(r)$ was 0.54 . Straight line is a regression line obtained by the least squares method.

inset) around the perimeter of the keratocyte or the minikeratocyte by the maximum intensity of the cell. The blue (keratocytes) and red (mini-keratocytes) lines in Fig. 3C almost overlap each other, suggesting that there was no difference in the distribution of the rear left and right focal adhesions between keratocytes and mini-keratocytes.

Next, we tested whether the area of focal adhesions was related to cell area, as was the case with the magnitude of the traction forces (Fig. 2E). The regions in which the fluorescence intensity of vinculin was greater than $30 \%$ of the maximum intensity in the keratocytes or mini-keratocytes were restricted to the rear left and right ends. We therefore regarded these as the regions of rear focal adhesion. We plotted the relationship between the area of the ventral region of the keratocytes or mini-keratocytes and the area of rear focal adhesion (Fig. 3D). The correlation coefficient $(r)$ between the two was 0.54 . There was a positive correlation between the area of the keratocytes or mini-keratocytes and the area of rear focal adhesion.

In keratocytes, stress fibers composed of actomyosin are positioned in such a way that they connect the rear left and right focal adhesions (Barnhart et al., 2011; Doyle and Lee, 2005; Lee and Jacobson, 1997). Contraction of these stress fibers is therefore thought to be one of the sources of the traction forces at the rear left and right ends. To test whether stress fibers were visible at the same position in keratocytes and mini-keratocytes, F-actin and myosin IIA were simultaneously stained in the keratocytes (Fig. 4A and $4 \mathrm{~B}$; typical images from 21 samples) and mini-keratocytes (Fig. 4C and 4D; typical images from 34 samples). Stress fibers (indicated by green arrows in Fig. 4A-4D) were seen not only in the keratocytes but also in the mini-keratocytes.

\section{Mechanism of deformation of the fan shape of keratocytes}

Blebbistatin, an inhibitor of myosin motor activity, induces fragmentation of keratocytes (Schaub et al., 2007). Treatment with low concentrations of blebbistatin induces lateral expansion of keratocytes and deforms the shape (Barnhart et al., 2011; Fuhs et al., 2014). To determine whether loss of the contractile force of actomyosin would shift the distribution of the traction forces and focal adhesions, we treated the keratocytes with blebbistatin. As seen by comparing the blebbistatin-treated cell (Fig. 5B) and -untreated cell (Fig. $5 \mathrm{E})$, keratocytes treated with blebbistatin expanded later- 

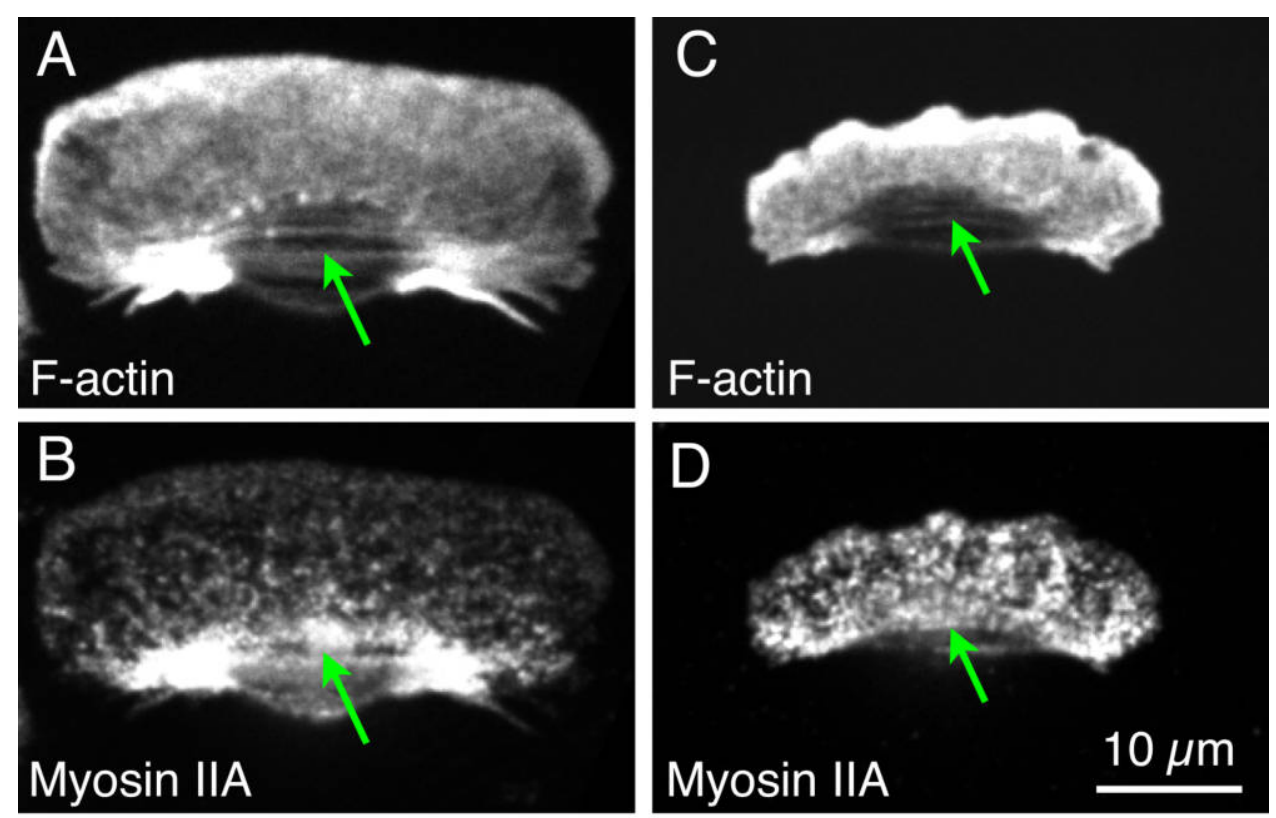

Kera

Mini

Fig. 4. Distribution of F-actin and myosin IIA in keratocytes and mini-keratocytes. (A) F-actin stained with Alexa Fluor phalloidin in a keratocyte. (B) Immunofluorescence staining of myosin IIA in the same sample as in A. (C) F-actin stained with Alexa Fluor phalloidin in a mini-keratocyte. (D) Immunofluorescence staining of myosin IIA in the same sample as in C. Green arrows indicate stress fibers. Images A and B, and C and D, are typical images from 21 and 34 samples, respectively.

ally (Fig. 5A and 5B; typical image from 35 samples). Although blebbistatin treatment did not change the curvature radius of keratocytes (compare left columns in Fig. 1H and Fig. 5A, $p=0.655$ ), it significantly increased center angle of them (compare right columns in Fig. $1 \mathrm{H}$ and Fig. $\left.5 \mathrm{~A}, p=2 \times 10^{-6}\right)$. Keratocytes treated with blebbistatin exerted only low traction forces at the rear left and right ends (Fig. 5C; typical image from 35 samples). Dots showing the relationship between the area of the ventral region of the blebbistatin-treated keratocytes and the maximum traction forces were added to Fig. 2E as blue triangles to produce Fig. 5D. The blue triangles are clearly out of the regression line of the keratocytes and mini-keratocytes without blebbistatin.

Next, we observed the distribution of F-actin, myosin IIA, and vinculin in blebbistatin-treated keratocytes (Fig. 6). F-actin and myosin IIA were simultaneously stained in the same samples (Fig. 6A and 6B; typical images from 49 samples). These figures indicate that the stress fibers were disrupted by blebbistatin treatment (no fiber can be seen at the tips of green arrows in Fig. 6A and 6B). Localization of vinculin at the left and right rear ends also decreased dramatically (indicated by green arrows in Fig. 6C; typical images from 25 samples). Dots showing the relationship between the area of the ventral region of the blebbistatintreated keratocytes and the region of rear focal adhesions were added to Fig. 3D as blue triangles to produce Fig.
$6 \mathrm{D})$. The blue triangles are clearly out of the regression line of the keratocytes and mini-keratocytes without blebbistatin. These results suggest that the fan shape of the keratocytes depends on the distributions of the traction forces and the focal adhesions.

\section{Discussion}

We revealed here that the shapes of the leading edges of keratocytes and mini-keratocytes were similar (Fig. 1). Mini-keratocytes, like keratocytes, exerted traction forces at the rear left and right ends (Fig. 2B and 2D). The magnitude of the traction forces and the area of the rear focal adhesions were proportional to the area of the ventral region of the keratocytes and mini-keratocytes (Fig. 2E and Fig. 3D). Blebbistatin decreased the focal adhesions and the forces at the rear left and right ends of the keratocytes and deformed the cells' fan shape (Fig. 5 and Fig. 6). These results suggest that the fan shape of keratocytes depends on optimal distribution of traction forces and that the magnitude of the traction forces depends on cell area. The optimal distribution of traction forces may make optimal tension balance of cell cortex to maintain the fan shape of keratocytes.

The magnitude of the traction forces should be determined by the number of stress fibers. Although the number 

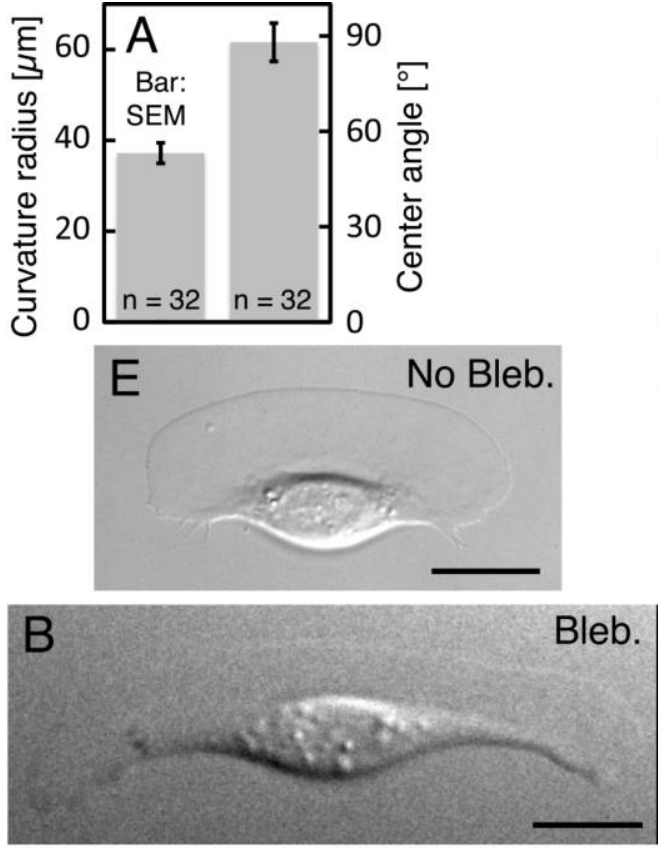

Bars, $10 \mu \mathrm{m}$
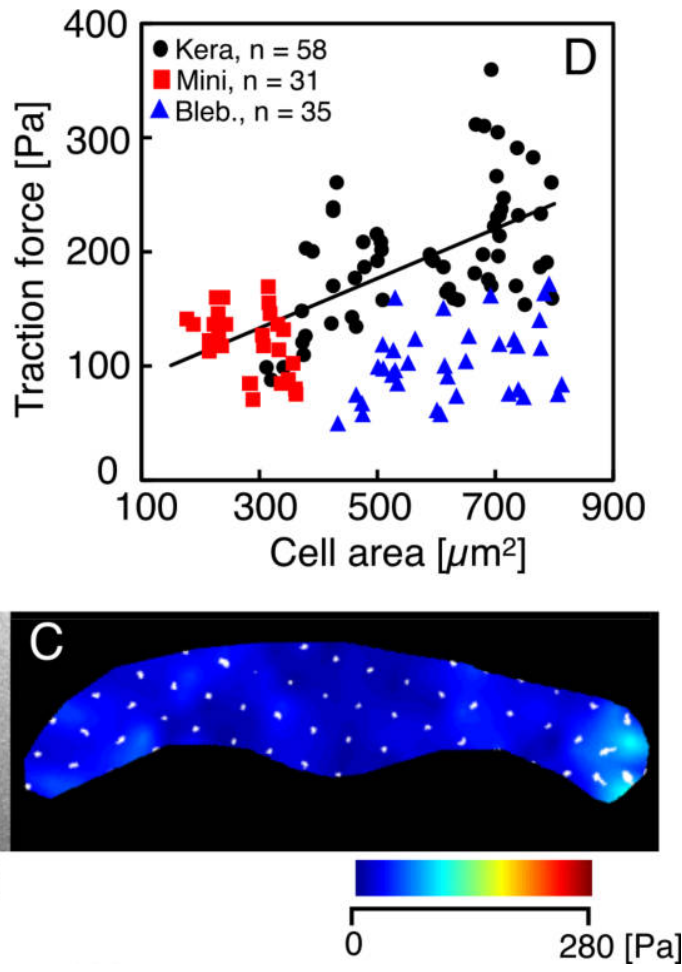

Fig. 5. Traction forces exerted by keratocytes treated with blebbistatin. (A) Averaged curvature radius (left) and center angle (right) of keratocytes treated with blebbistatin. (B) DIC image of a blebbistatin-treated keratocyte. (C) Traction forces of the keratocyte in B. Direction and length of white arrows in C respectively indicate the direction and the relative magnitude of traction forces at the base of the arrows. (D) Relationship between the area of the ventral region of the blebbistatin-treated keratocytes and the maximum traction force (blue triangles) has been added to Fig. $2 \mathrm{E}$ to produce this graph. (E) DIC image of a blebbistatin-untreated keratocyte for comparison with B. Images B and C, and E, are typical images from 35 and 121 samples, respectively.

was not measured directly in this paper, the area of rear focal adhesion which connect the stress fibers (Barnhart et al., 2011; Doyle and Lee, 2005; Lee and Jacobson, 1997) was also proportional to the ventral area of the keratocytes (Fig. 3D). Upon cell divisions, the dividing cell seems to generate optimal number of stress fibers to exert optimal forces dependent on the cell area via transcription of gene upon cell divisions. However, even after the fragmentation, the magnitude of the traction forces (Fig. 2E) and the area of the rear focal adhesions (Fig. 3D) were proportional to the cell area, suggesting that keratocytes should redistribute the stress fibers by the appropriate number upon the fragmentation. How they realize such re-distribution of the stress fibers is yet mystery. The physiological significance of the positive relationship between the ventral area and the force of keratocytes is also unknown. In most of migrating cell types, the traction forces at the rear end are the result of contraction of the rear, which is indispensable for advance (Iwadate and Yumura, 2008a; Munevar et al., 2001a, 2001b; Shin et al., 2010). The positive relationship may be necessary for the same migration velocity in different sizes of the cell (Fig. 2F).

The other remaining question of what generates the opti- mal distribution of traction forces is also interesting. Local activation of actomyosin by the application of calyculin A, an inhibitor of myosin light-chain phosphatase, to discoid stationary keratocytes makes them fan-shaped and initiates motility directed away from the site of stimulation (Yam et al., 2007). In addition, local transient mechanical stimulus to the discoid stationary lamellar fragments induces the anisotropic formation of actomyosin bundles at the stimulated edge, and the bundles are sustained even after withdrawal of the stimulus (Verkhovsky et al., 1999). These findings suggest that the anterior-posterior polarity in keratocytes is generated by a positive-feedback loop intrinsic to the actinmyosin II machinery. Redistribution of traction forces should occur simultaneously with, or just after, polarity generation. The actomyosin bundles may adhere to the substratum at both ends, which become focal adhesions. They then begin to exert traction forces as stress fibers.

How the distribution of traction forces regulates the shape of the leading edge is another interesting question. The plasma membrane tension of keratocytes is not uniform throughout the cell (Lieber et al., 2013, 2015). It is thus possible that the membrane tension around the rear left and right ends, where high traction forces are exerted, is 

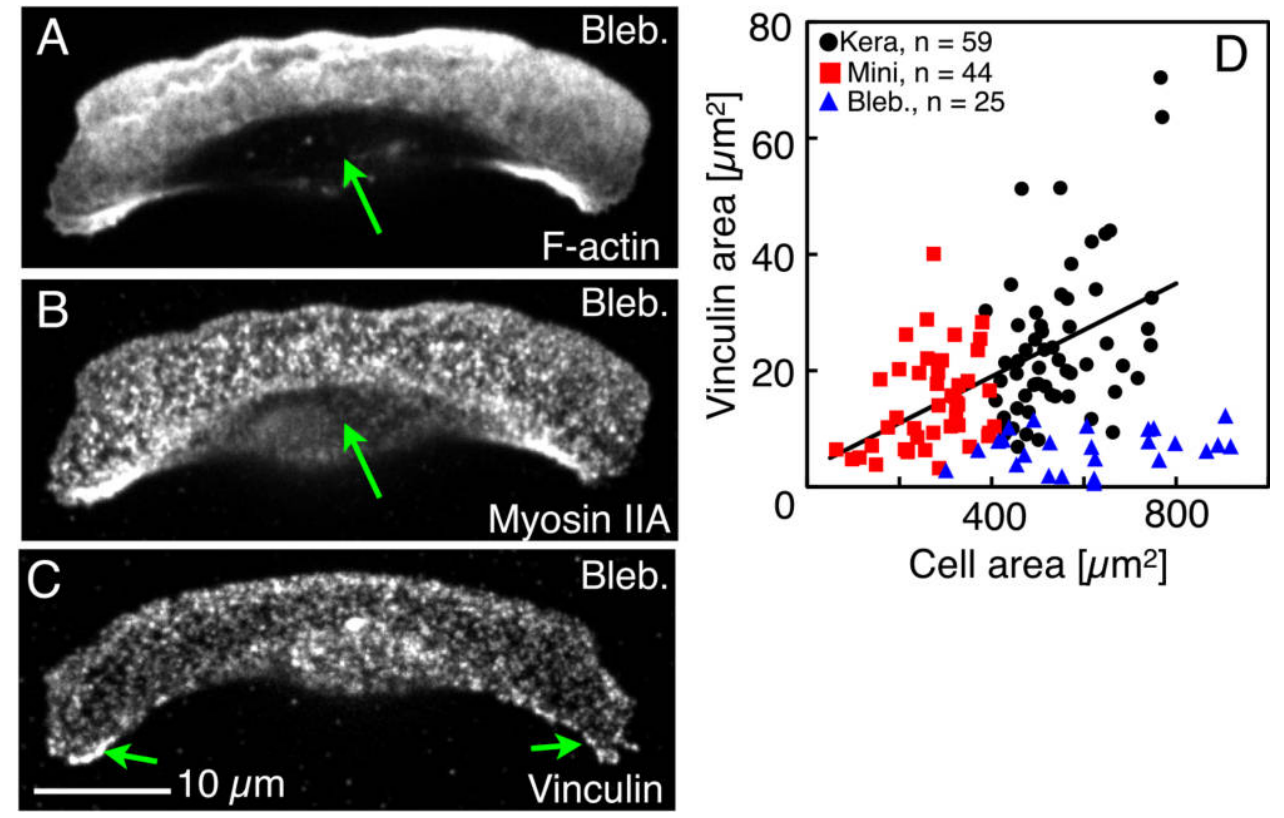

Cell area $\left[\mu \mathrm{m}^{2}\right]$

Fig. 6. Distribution of F-actin, myosin IIA, and vinculin in blebbistatin-treated keratocytes. (A) F-actin stained with Alexa Fluor phalloidin in a keratocyte. (B) Immunofluorescence staining of myosin IIA in the same sample as in A. Images A and B are typical images from 49 samples. (C) Immunofluorescence staining of vinculin. The image is a typical one from 25 samples. Green arrows in A and B, and C, indicate "no" stress fibers and slightly localized vinculin, respectively. (D) Relationship between the area of the ventral region of the blebbistatin-treated keratocytes and the region of rear focal adhesions (blue triangles) has been added to Fig. 3D to produce this graph.

higher than that at the center of the leading edge. This graded membrane tension may induce not only a graded RF rate but also a graded actin-polymerization rate, because the rise in membrane tension inhibits the actin-polymerization rate (Tsujita et al., 2015).

In fibroblasts, the distance from the cell center to the perimeter controls traction forces at the perimeter by regulating the area of focal adhesions there (Rape et al., 2011). When the distance is too long or too short, only small focal adhesions are generated and only weak traction forces are exerted. There is an optimal distance for properly generating focal adhesions and exerting traction forces. This suggests that the cell shape regulates the traction forces, and that the traction forces in turn regulate the cell shape. It is thus likely that the distribution of traction forces and the shape of the cell control each other through the formation of focal adhesions. Keratocytes cannot form the typical fan shape on substrata with low or high levels of adhesion (Barnhart et al., 2011); optimal adhesiveness of the substratum is therefore required to generate and maintain the fan shape. To resolve the remaining above-mentioned issues, the interactions among cell shape, plasma membrane tension, substratum adhesiveness, and traction forces need to be investigated in detail in a future study.
Acknowledgments. We thank Dr. H. Miyoshi (Riken, Wako, Japan) for instruction in cell fixation and staining, Dr. T. Mizuno (AIST, Tsukuba, Japan) for instruction in live cell preparation, and T. Nakata and $H$. Nakashima (Yamaguchi Univ.) for help with performing the experiments. Y.I. was supported by MEXT Kakenhi Grants Nos. 26103524, 26650050, and $15 \mathrm{H} 01323$.

\section{Author contributions}

C.O. and Y.I. designed the experiments. A.S. and C.O. performed the experiments. C.O. and Y.I. wrote the manuscript.

\section{References}

Barnhart, E.L., Allen, G.M., Jülicher, F., and Theriot, J.A. 2010. Bipedal locomotion in crawling cells. Biophys. J., 98: 933-942.

Barnhart, E.L., Lee, K.-C., Keren, K., Mogilner, A., and Theriot, J.A. 2011. An adhesion-dependent switch between mechanisms that determine motile cell shape. PLoS Biol., 9: e1001059.

Beningo, K.A., Dembo, M., Kaverina, I., Small, J.V., and Wang, Y.L. 2001. Nascent focal adhesions are responsible for the generation of strong propulsive forces in migrating fibroblasts. J. Cell Biol., 153: 881-888.

Burton, K., Park, J.H., and Taylor, D.L. 1999. Keratocytes generate traction forces in two phases. Mol. Biol. Cell, 10: 3745-3769.

Chen, Z., Lessey, E., Berginski, M.E., Cao, L., Li, J., Trepat, X., Itano, M., Gomez, S.M., Kapustina, M., Huang, C., et al. 2013. Gleevec, an Abl family inhibitor, produces a profound change in cell shape and migra- 
tion. PLoS ONE, 8: e52233.

Dembo, M., Oliver, T., Ishihara, A., and Jacobson, K. 1996. Imaging the traction stresses exerted by locomoting cells with the elastic substratum method. Biophys. J., 70: 2008-2022.

Dembo, M. and Wang, Y.L. 1999. Stresses at the cell-to-substrate interface during locomotion of fibroblasts. Biophys. J., 76: 2307-2316.

Doyle, A., Marganski, W., and Lee, J. 2004. Calcium transients induce spatially coordinated increases in traction force during the movement of fish keratocytes. J. Cell Sci., 117: 2203-2214.

Doyle, A.D. and Lee, J. 2005. Cyclic changes in keratocyte speed and traction stress arise from $\mathrm{Ca}^{2+}$-dependent regulation of cell adhesiveness. J. Cell Sci., 118: 369-379.

Euteneuer, U. and Schliwa, M. 1984. Persistent, directional motility of cells and cytoplasmic fragments in the absence of microtubules. Nature, 310: 58-61.

Forscher, P. and Smith, S.J. 1988. Actions of cytochalasins on the organization of actin filaments and microtubules in a neuronal growth cone. $J$. Cell Biol., 107: 1505-1516.

Fournier, M.F., Sauser, R., Ambrosi, D., Meister, J.-J., and Verkhovsky, A.B. 2010. Force transmission in migrating cells. J. Cell Biol., 188: 287-297.

Friedl, P. and Alexander, S. 2011. Cancer invasion and the microenvironment: plasticity and reciprocity. Cell, 147: 992-1009.

Fuhs, T., Goegler, M., Brunner, C.A., Wolgemuth, C.W., and Kaes, J.A. 2014. Causes of retrograde flow in fish keratocytes. Cytoskeleton, $\mathbf{7 1}$ 24-35.

Galbraith, C.G., Yamada, K.M., and Sheetz, M.P. 2002. The relationship between force and focal complex development. J. Cell Biol., 159: 695705 .

Gupton, S.L. and Waterman-Storer, C.M. 2006. Spatiotemporal feedback between actomyosin and focal-adhesion systems optimizes rapid cell migration. Cell, 125: 1361-1374.

Harris, A.K., Wild, P., and Stopak, D. 1980. Silicone rubber substrata: a new wrinkle in the study of cell locomotion. Science, 208: 177-179.

Harris, A.K., Stopak, D., and Wild, P. 1981. Fibroblast traction as a mechanism for collagen morphogenesis. Nature, 290: 249-251.

Iwadate, Y. and Yumura, S. 2008a. Actin-based propulsive forces and myosin-II-based contractile forces in migrating Dictyostelium cells. J. Cell Sci., 121: 1314-1324.

Iwadate, Y. and Yumura, S. 2008b. Molecular dynamics and forces of a motile cell simultaneously visualized by TIRF and force microscopies. BioTechniques, 44: 739-750.

Jurado, C., Haserick, J.R., and Lee, J. 2005. Slipping or gripping? Fluorescent speckle microscopy in fish keratocytes reveals two different mechanisms for generating a retrograde flow of actin. Mol. Biol. Cell, 16: $507-518$.

Katoh, K., Hammar, K., Smith, P.J., and Oldenbourg, R. 1999a. Birefringence imaging directly reveals architectural dynamics of filamentous actin in living growth cones. Mol. Biol. Cell, 10: 197-210.

Katoh, K., Hammar, K., Smith, P.J., and Oldenbourg, R. 1999b. Arrangement of radial actin bundles in the growth cone of Aplysia bag cell neurons shows the immediate past history of filopodial behavior. Proc. Natl. Acad. Sci. USA, 96: 7928-7931.

Keren, K., Pincus, Z., Allen, G.M., Barnhart, E.L., Marriott, G., Mogilner A., and Theriot, J.A. 2008. Mechanism of shape determination in motile cells. Nature, 453: 475-480.

Lauffenburger, D.A. and Horwitz, A.F. 1996. Cell migration: a physically integrated molecular process. Cell, 84: 359-369.

Lee, J., Ishihara, A., Theriot, J.A., and Jacobson, K. 1993. Principles of locomotion for simple-shaped cells. Nature, 362: 167-171.

Lee, J. and Jacobson, K. 1997. The composition and dynamics of cellsubstratum adhesions in locomoting fish keratocytes. J. Cell Sci., 110: 2833-2844.
Lieber, A.D., Yehudai-Resheff, S., Barnhart, E.L., Theriot, J.A., and Keren, K. 2013. Membrane tension in rapidly moving cells is determined by cytoskeletal forces. Curr. Biol., 23: 1409-1417.

Lieber, A.D., Schweitzer, Y., Kozlov, M.M., and Keren, K. 2015. Frontto-rear membrane tension gradient in rapidly moving cells. Biophys. J., 108: 1599-1603.

Lo, C.M., Wang, H.B., Dembo, M., and Wang, Y.L. 2000. Cell movement is guided by the rigidity of the substrate. Biophys. J., 79: 144-152.

Lombardi, M.L., Knecht, D.A., Dembo, M., and Lee, J. 2007. Traction force microscopy in Dictyostelium reveals distinct roles for myosin II motor and actin-crosslinking activity in polarized cell movement. $J$. Cell Sci., 120: 1624-1634.

Martin, P. 1997. Wound healing-aiming for perfect skin regeneration. Science, 276: 75-81.

Miyoshi, H. and Adachi, T. 2012. Spatiotemporal coordinated hierarchical properties of cellular protrusion revealed by multiscale analysis. Integr. Biol., 4: 875-888.

Mizuno, T., Sakai, T., Yoshioka, K. and Kawasaki, K. 2003. Differencefrom-prediction imaging for high resolution shape analysis of locomoting cells. Bioimages, 11: 61-66.

Mizuno, T. and Sekiguchi, Y. 2011. Staurosporine induces lamellipodial widening in locomoting fish keratocytes by abolishing the gradient from radial extension of leading edge. Biophysics, 7: 69-75.

Mogilner, A. and Keren, K. 2009. The shape of motile cells. Curr. Biol., 19: R762-R771.

Munevar, S., Wang, Y., and Dembo, M. 2001a. Traction force microscopy of migrating normal and H-ras transformed $3 \mathrm{~T} 3$ fibroblasts. Biophys. J., 80: $1744-1757$.

Munevar, S., Wang, Y.L., and Dembo, M. 2001b. Distinct roles of frontal and rear cell-substrate adhesions in fibroblast migration. Mol. Biol. Cell, 12: 3947-3954.

Munevar, S., Wang, Y.-L., and Dembo, M. 2004. Regulation of mechanical interactions between fibroblasts and the substratum by stretchactivated $\mathrm{Ca}^{2+}$ entry. J. Cell Sci., 117: 85-92.

Okeyo, K.O., Adachi, T., Sunaga, J., and Hojo, M. 2009. Actomyosin contractility spatiotemporally regulates actin network dynamics in migrating cells. J. Biomech., 42: 2540-2548.

Parent, C.A. 2004. Making all the right moves: chemotaxis in neutrophils and Dictyostelium. Curr. Opin. Cell Biol., 16: 4-13.

Rape, A.D., Guo, W.-H., and Wang, Y.-L. 2011. The regulation of traction force in relation to cell shape and focal adhesions. Biomaterials, 32: 2043-2051.

Ridley, A.J., Schwartz, M.A., Burridge, K., Firtel, R.A., Ginsberg, M.H., Borisy, G., Parsons, J.T., and Horwitz, A.R. 2003. Cell migration: integrating signals from front to back. Science, 302: 1704-1709.

Schaub, S., Bohnet, S., Laurent, V.M., Meister, J.-J., and Verkhovsky, A.B. 2007. Comparative maps of motion and assembly of filamentous actin and myosin II in migrating cells. Mol. Biol. Cell, 18: 3723-3732.

Shin, M.E., He, Y., Li, D., Na, S., Chowdhury, F., Poh, Y.-C., Collin, O., $\mathrm{Su}$, P., de Lanerolle, P., Schwartz, M.A., et al. 2010. Spatiotemporal organization, regulation, and functions of tractions during neutrophil chemotaxis. Blood, 116: 3297-3310.

Tanimoto, H. and Sano, M. 2014. A simple force-motion relation for migrating cells revealed by multipole analysis of traction stress. Biophys. J., 106: 16-25.

Tseng, Q., Duchemin-Pelletier, E., Deshiere, A., Balland, M., Guillou, H., Filhol, O., and Théry, M. 2012. Spatial organization of the extracellular matrix regulates cell-cell junction positioning. Proc. Natl. Acad. Sci. USA, 109: 1506-1511.

Tsugiyama, H., Okimura, C., Mizuno, T., and Iwadate, Y. 2013. Electroporation of adherent cells with low sample volumes on a microscope stage. J. Exp. Biol., 216: 3591-3598.

Tsujita, K., Takenawa, T., and Itoh, T. 2015. Feedback regulation between 
plasma membrane tension and membrane-bending proteins organizes cell polarity during leading edge formation. Nat. Cell Biol., doi: $10.1038 /$ ncb3162.

Vallotton, P., Danuser, G., Bohnet, S., Meister, J.-J., and Verkhovsky, A.B. 2005. Tracking retrograde flow in keratocytes: news from the front. Mol. Biol. Cell, 16: 1223-1231.

Verkhovsky, A.B., Svitkina, T.M., and Borisy, G.G. 1999. Selfpolarization and directional motility of cytoplasm. Curr. Biol., 9: 11-20.
Yam, P.T., Wilson, C.A., Ji, L., Hebert, B., Barnhart, E.L., Dye, N.A., Wiseman, P.W., Danuser, G., and Theriot, J.A. 2007. Actin-myosin network reorganization breaks symmetry at the cell rear to spontaneously initiate polarized cell motility. J. Cell Biol., 178: 1207-1221.

(Received for publication, August 3, 2015, accepted, December 22, 2015 and published online, January 9, 2016) 